Elsevier

\title{
Function-limiting dysesthetic pain syndrome among traumatic spinal cord injury patients: a cross-sectional study
}

\author{
Gary Davidoff *, Elliot Roth **, Mary Guarracini **, James Sliwa ** \\ and Gary Yarkony ** \\ * Department of Physical Medicine and Rehabilitation, University of Michigan Medical Center, Ann Arbor, \\ MI 48109 (U.S.A.), and ** Department of Rehabilitation Medicine, Northwestern University \\ Medical School, Chicago, IL 60611 (U.S.A.)
}

(Received 3 March 1986, revised received 17 June 1986, accepted 29 August 1986)

\begin{abstract}
Summary Diffuse burning dysesthetic sensations distal to the level of spinal injury are the most common and disabling painful sequelae of traumatic spinal cord injury (SCI). In a cross-sectional study of $19 \mathrm{SCI}$ patients, clinical characteristics and results of 3 validated pain measurement instruments (McGill Pain Questionnaire, Sternbach Pain Intensity and Zung Pain and Distress Scale) were used to develop a profile of function-limiting dysesthetic pain sydrome (DPS). Compared to a cohort of 147 patients admitted to the Midwest Regional Spinal Cord Injury Care System during the time period of the study, subjects were more likely to have paraplegia, incomplete sensory myelopathy, gunshot wounds to the spine and non-surgical spinal stabilization. Most patients described the pain as 'cutting, 'burning,' 'piercing,' 'radiating' and 'tight.' The majority of patients located the pain internally and in the lower extremities. Values obtained from 6 McGill Pain Questionnaire subscales, 2 Sternbach Pain Intensity ratings and the Zung Pain and Distress index equalled or exceeded those reported for other pain syndromes. Use of these validated pain measures resulted in a systematic comprehensive assessment of function-limiting DPS following SCI.
\end{abstract}

Key words: Spinal cord injury; Pain; Pain measures

\section{Introduction}

Painful spontaneous sensations are frequent yet enigmatic sequelae of traumatic spinal cord injury (SCI), with an estimated incidence of $11-94 \%[5,6,11,24,25,34$, $35,37,49]$. Although severe disabling pain is noted less often than mild incidental

Correspondence to: Gary Davidoff, M.D., Department of Physical Medicine and Rehabilitation, Box 0042, University Hospital, Ann Arbor, MI 48109, U.S.A. 
pain, many SCI patients report some interference with daily function secondary to their discomfort $[5,11,24,30,34,37,39]$. A variety of classification systems has been proposed $[3,6,7,10,12,15,18,22,24,27,30,32,35,38]$. Most authors agree that the diffuse dysesthetic pain which occurs distal to the level of spinal injury is the most common of these syndromes $[7,12,37,38]$.

A number of investigators have provided clinicians with descriptions of the dysesthetic pain syndrome $[3-7,10-12,15,18,22,27,32,37-39,41,47]$. Based upon patient reports, common features have included unpleasant sensations of burning, stinging or stabbing quality associated with disturbed sensibility. Pain occurs below the level of spinal injury in a diffuse non-radicular pattern. Presentation usually occurs within 1 year of injury. The influence of etiology, level and completeness of spinal injury upon the development of DPS has been controversial. Many authors have reported their experiences with pharmacologic, psychologic, physical and surgical methods of treatment; response to these interventions has varied $[2,3,5,7,8,12-14,16,18,21,23-25,27,28,35-37,39,42,43,49]$. Proposed mechanisms have directed attention to central or spinal sites of origin $[15,30]$, but more precise information concerning pathogenesis has not yet been elucidated.

Limitations in the ability to quantify clinically significant characteristics of pain have precluded adequate objective evaluation of DPS. The present study was undertaken to systematically examine the disabling diffuse dysesthetic pain syndrome experienced by SCI patients. Use of clinical data and results obtained from 3 validated pain assessment instruments provided an objective profile of the quality and magnitude of this pain syndrome.

\section{Methods}

\section{Subjects}

The study group consisted of spinal cord injury (SCI) patients who completed the first phase of a randomized, double-blind, placebo-controlled study of a pharmacologic agent, trazodone hydrochloride, for the treatment of function-limiting DPS. Following orientation of attending physicians, house officers and members of the nursing staff at the Rehabilitation Institute of Chicago (RIC), Northwestern University Medical School, Chicago, IL, patients were recruited for participation in this research project. All potential subjects were referred to one of the physician-investigators (E.R.), who then performed preliminary telephone screening evaluations. Patients who met study criteria were invited to participate in the evaluation protocol. All participants gave informed written consent. This study was approved by the Institutional Review Board of Northwestern University Medical School, Chicago, IL.

Only patients with traumatic myelopathy were studied. Exclusion criteria included age less than 18 years, lack of fluency with the English language and history of recent ethanol or substance abuse. All participants met the following criteria:

(1) A history of dysesthetic pain of at least 1 month duration, with initial onset during the first post-injury year; 
(2) Failure to respond to conventional treatments, including therapeutic exercise, physical modalities, non-prescription analgesic medications, non-steroidal anti-inflammatory agents or narcotics; and

(3) Pain-induced functional impairment, defined as the presence of one or more of the following symptoms: (a) disturbance of sleep-wake cycle, (b) inability to perform self-care activities, or (c) inability to fully comply with a therapeutic exercise program.

\section{Procedure}

Each patient underwent a comprehensive interview in which the history of the pain syndrome was reviewed in detail. Pertinent data included: age, sex, duration of pain, etiology, level and completeness of injury, history of surgical spinal stabilization, previous diagnostic evaluations, past treatment modalities and associated medical conditions. A thorough neurologic and general physical examination was performed on each patient. Determinations of motor and sensory levels and degree of completeness of injury were made according to American Spinal Injury Association criteria [1].

Following history and physical examination, each patient completed 3 validated pain measurement instruments. These measures consisted of the following:

(1) McGill Pain Questionnaire (MPQ). The MPQ uses word descriptors to generate information concerning affective, sensory and evaluative aspects of pain. Three types of measures are obtained from the MPQ: (a) the pain rating index (PRI) is based upon the rank values of words selected from a 20-item inventory to describe the sensory (PRI-S), affective (PRI-A), evaluative (PRI-E) and total (PRI-T) aspects of pain; (b) the number of words chosen (NWC) from the same 20-category word list is recorded; (c) the present pain intensity (PPI) is an indicator of the severity of pain, on a scale from 1 to 5 , experienced by the patient at the time of assessment. The MPQ includes a series of questions concerning pain location, exacerbating factors and methods of relief. Many authors $[9,17,19,20,29,40,46]$ have reported the MPQ to be a valid, reliable and useful instrument for pain assessment. In this study, the MPQ was administered to each patient by an investigator or a trained research assistant because interview format has been shown to enhance the sensitivity of the MPQ subscales [26].

(2) Sternbach Pain Intensity (SPI). The SPI requires each patient to rank severity of his or her pain on a numerical scale from 0 to 100. SPI defines 0 as no pain and 100 as pain so severe that the patient would commit suicide. In the present study, patients were asked to provide a numerical rating of their pain for the day of assessment (SPI-day) and for the week preceding evaluation (SPI-week). Sternbach and co-workers $[44,45]$ demonstrated the validity of this scale in neurogenic and myofascial pain syndromes.

(3) Zung Pain and Distress Index (PAD). The PAD is a 20-item questionnaire designed for self-administration. Each patient responds by indicating the extent to which he or she agrees with statements describing mood and behavioral changes associated with pain. Zung [50] demonstrated the PAD to be a robust measure of pain and accompanying emotional distress. 


\section{Data analysis}

The frequency distributions of pertinent patient characteristics were determined. Important clinical parameters included: age, etiology, level and completeness of spinal injury, history of surgical spinal stabilization, presence of spasticity, duration of pain, previous diagnostic evaluations and past therapeutic regimens. These data were compared to the frequency distributions of characteristics of all patients with acute SCI admitted to the Midwest Regional Spinal Cord Injury Care System (MRSCICS) of Northwestern University Medical School, Chicago, IL, between July 1. 1984 and June 30, 1985 [33]. These comparisons were felt to be justified because findings of the National SCI Data Research Center [48] indicated that incidence rates and clinical features of the MRSCICS patient group included in the present investigation were similar to those of the general population of SCI patients in the United States. During the study period, 147 patients were admitted to the MRSCICS for initial care of acute SCI. Significant differences between certain characteristics of the study group and those of the MRSCICS registry patients were determined using a normal approximation of the binomial distribution to obtain confidence intervals.

Means and standard errors of the means were calculated for each of the $6 \mathrm{MPQ}$ subscales, the 2 SPI ratings and the PAD index. The degree of association between pain scales and 5 clinical characteristics (etiology, completeness and level of injury, presence of spasticity and surgical stabilization) was determined using Student's $t$ test. Simple linear regression analysis evaluated the relationship between pain ratings and duration of symptoms. Evaluation of intercorrelations between MPQ subscales, SPI ratings and the PAD index was also performed using simple linear regression analysis. Statistical analyses were performed utilizing the Michigan Interactive Data Analysis System (MIDAS) of the University of Michigan Computer Center.

\section{Results}

\section{Patient characteristics}

Nineteen patients with disabling dysesthetic pain syndrome (DPS) were evaluated. Mean age was 36.6 years with a range between 25 and 69 years. Seventeen (90\%) were male. Three (16\%) patients were quadriplegic. Of the 19 subjects, five $(26 \%)$ sustained complete injuries. Spasticity was noted in eight (42\%). Etiologies of injury, in descending order of frequency, were: gunshot wounds $(52.6 \%)$; falls $(21.1 \%)$; road traffic accidents (10.5\%); sporting accidents (5.3\%); and other causes (10.5\%). Six (34\%) patients underwent surgical stabilization of the spine. At the time of evaluation, duration of symptoms averaged (mean \pm standard error of mean) $46.8 \pm 10.2$ months. Significant differences between the frequency distributions of certain clinical characteristics and those of the MRSCICS patient cohort were identified. Patients with DPS were more likely to have: paraplegia $(P<0.01)$, incomplete sensory myelopathy $(P<0.05)$ and SCI resulting from gunshot wounds $(P<0.01)$. Study participants were less likely to have undergone surgical spinal stabilization 
TABLE I

PATIENT CHARACTERISTICS

\begin{tabular}{llll}
\hline Item & DPS patients & MRSCICS patients & Significance \\
\hline Number & 19 & 147 & \\
Quadriplegic & $15.8 \%$ & $54.4 \%$ & $P<0.01$ \\
Complete sensory & $26.3 \%$ & $48.6 \%$ & $P<0.05$ \\
Surgical stabilization & $33.6 \%$ & $66.4 \%$ & $P<0.01$ \\
Etiology & & & \\
$\quad$ Gunshot wound & $52.6 \%$ & $15.7 \%$ & $P<0.01$ \\
$\quad$ Falls & $21.1 \%$ & $27.2 \%$ & \\
Road traffic accidents & $10.5 \%$ & $32.7 \%$ & \\
Other & $10.5 \%$ & $12.9 \%$ & \\
Sports incidents & $5.3 \%$ & $11.6 \%$ & \\
Age (mean) & 36.6 years & 32.0 years & \\
Duration of pain (mean) & $46.8 \pm 10.2$ months & & \\
Spasticity present & $42 \%$ & & \\
\hline
\end{tabular}

$(P<0.01)$ than their MRSCICS counterparts. These data are summarized in Table I.

\section{Previous diagnostic and therapeutic management}

Most patients underwent at least one diagnostic procedure to evaluate the pain syndrome prior to entry into the study. These included: electromyography $(42 \%)$, plain radiography $(42 \%)$, myelography $(21 \%)$, computed spine tomography $(21 \%)$, diagnostic peripheral nerve block (21\%), contrast radiographic studies of the gastrointestinal tract $(16 \%)$ and radionuclide bone scanning $(11 \%)$. None of these studies demonstrated abnormal findings other than those attributable to sequelae of traumatic SCI.

All patients had been previously treated with a variety of pharmacologic and non-pharmacologic interventions. Eight (42\%) patients reported use of a transcutaneous nerve stimulator. Two (10.5\%) were given biofeedback training and one (5\%) received acupuncture treatment. Nine (47\%) patients had previously been treated with aspirin or acetaminophen, but only three $(16 \%)$ had received non-steroidal anti-inflammatory agents. The majority $(74 \%)$ of patients had taken narcotics for their pain. Neuroleptic medications were used by three (16\%) and tricyclic antidepressant medications by ten $(53 \%$; amitriptyline $=9$, doxepin $=1)$. Four $(21 \%)$ patients were given a trial of phenytoin and three (16\%) had used carbamazepine. Only one (5\%) patient received a therapeutic peripheral nerve block and two (10.5\%) underwent surgical implantation of an epidural stimulator. None of these interventions resulted in consistent or lasting relief.

\section{Pain assessment}

The words most frequently chosen from the MPQ list were: 'cutting' (63\%), 'burning' (58\%), 'piercing' (47\%), 'radiating' (47\%), 'tight' (37\%), 'cruel' (37\%) and 
TABLE II

PAIN MEASURES OF DPS PATIENTS

\begin{tabular}{lcc}
\hline Item & Mean & S.E.M. \\
\hline PRI-T & 32.9 & 2.3 \\
PRI-S & 23.7 & 1.7 \\
PRI-A & 7.1 & 1.0 \\
PRI-F & 2.6 & 0.3 \\
PPI & 2.1 & 0.2 \\
NWC & 13.4 & 1.0 \\
SPI-day & 60.6 & 5.4 \\
SPI-week & 70.5 & 4.2 \\
PAD & 59.6 & 2.8
\end{tabular}

'nagging' (37\%). Of this list, sensory components of pain were represented by 4 words, affective components by 2 words and evaluative components by 1 word. Sixteen $(84 \%)$ of the patients described the pain as 'internal' and three (16\%) described it as 'mixed internal and external.' All pain sensations were distal to the level of spinal injury. The most common locations were: lower extremities (84\%), posterior trunk $(63 \%)$, anterior trunk $(42 \%)$ and upper extremities $(16 \% ; 100 \%$ of the quadriplegic patients).

Values of the $6 \mathrm{MPQ}$ subscales, 2 SPI scores and PAD index are listed in Table II. There were no significant relationships between each of the 9 pain measures and each of 5 clinical characteristics, including paraplegia, completeness of injury, presence of spasticity, gunshot wound etiology and surgical spinal stabilization. Simple linear regression analysis failed to demonstrate a significant correlation between the pain scales and duration of symptoms.

TABLE III

LINEAR REGRESSION ANALYSIS OF DPS PATIENT PAIN MEASURES $\left(R^{2}\right)$

\begin{tabular}{lllllllll}
\hline & PRI-T & PRI-S & PRI-A & PRI-E & PPI & NWC & SPI-day & SPI-week \\
\hline PRI-T & - & - & - & - & - & - & - & - \\
PRI-S & N.S. & - & - & - & - & - & - & - \\
PRI-A & $0.369^{b}$ & N.S. & - & - & - & - & - & - \\
PRI-E & N.S. & N.S. & N.S. & - & - & - & - & - \\
PPI & N.S. & N.S. & N.S. & N.S. & - & - & - & - \\
NWC & $0.783^{c}$ & N.S. & $0.296^{\text {a }}$ & N.S. & N.S. & - & - & - \\
SPI-day & N.S. & N.S. & N.S. & N.S. & N.S. & N.S. & - & - \\
SPI-week & N.S. & N.S. & N.S. & N.S. & N.S. & N.S. & $0.750^{c}$ & - \\
PAD & N.S. & N.S. & N.S. & N.S. & N.S. & $0.230^{\text {a }}$ & N.S. & N.S. \\
\hline
\end{tabular}

N.S. $=$ not significant.

a $P<0.05$.
b $P<0.01$.
" $P<0.001$. 
Table III demonstrates intercorrelations between each of the pain scales using simple linear regression analysis. A strong correlation between PRI-T and NWC was noted $(P<0.001)$. NWC accounted for $81 \%$ of the variance in PRI-T. Correlations were also noted of PRI-A with PRI-T $(P<0.01)$ and of PRI-A with NWC $(P<0.05)$. There was a strong relationship between the Sternbach Pain Intensity ratings evaluated for the day of assessment (SPI-day) and for the week prior to assessment (SPI-week) $(P<0.001)$. A correlation was noted between PAD index and NWC $(P<0.05)$. No other significant intercorrelations between pain scales were observed.

\section{Discussion}

The incidence of pain following traumatic SCI ranges between 11 and $94 \%$ $[5,6,11,24,25,34,35,37,49]$. Davis and Martin [11] studied 471 patients with SCI, over $90 \%$ of whom complained of diffuse burning pains. Similar figures were found by Bors [4], Botterell et al. [5] and Pollack et al. [38]. Munro [34] minimized the clinical importance of pain in SCI. Burke [6] reported that the incidence of pain following SCI differed according to treatment center. Reports of degree of pain-induced functional decrement among SCI patients vary. It is generally agreed that $10-33 \%$ of patients require medical or surgical intervention because of the severity of their pain $[5,11,24,34,37,39]$. Indeed, one survey [37] revealed that one-fourth of selected patients would trade neurologic recovery, continence or sexual function for pain relief.

Many of these studies are limited by a lack of discrimination between pain syndrome types. A number of categories and descriptions of SCI pain have been reported $[3,6,7,10,12,15,18,22,24,27,30,32,35,38]$. Burke [6] classified pain into 3 types: localized (radicular), visceral and diffuse (causalgia). The most recent schema was developed by Donovan et al. [15], who included the following categories: cauda equina (radicular), visceral, mechanical (myofascial), psychic and spinal cord (dysesthetic). Patients in the present investigation complained of diffuse causalgia or dysesthetic sensations distal to the level of spinal injury.

The purpose of this study was to objectively evaluate characteristics and pain descriptions of a group of traumatic SCI patients suffering from function-limiting dysesthetic pain syndrome (DPS). Adherence to strict inclusion criteria provided a homogeneous sample to study. The majority of patients had incomplete sensory injuries, were paraplegic and were gunshot wound victims. Most did not undergo surgical spinal stabilization. In contrast, Burke's findings [6] suggested that surgical stabilization was associated with an increased incidence of pain, but that etiology exerted no influence upon the development of the pain syndrome. Richards et al. [41] demonstrated that the frequency of pain was independent of level, etiology and surgical treatment. Additional conflicting descriptions of clinical characteristics associated with an increased incidence of pain in SCI patients were reported by Botterell et al. [5], Davis and Martin [11] and Waisbrod et al. [47]. Results of these studies differ from the findings of the present investigation, and from each other, in 
part. because of failure to distinguish between DPS and other types of painful sequelae of SCI.

In this study, evaluation of MPQ pain descriptions indicated that nearly all patients chose 1 or more of 5 words (cutting, burning, piercing, radiating, tight). These descriptions were similar to those selected by the patients of Waisbrod et al. [47]. Sixteen patients described the pain as 'internal' and three felt that the pain was of 'mixed internal and external' quality. All patients complained of dysesthesias distal to the level of spinal injury; $84 \%$ had lower extremity pain. These findings confirm that this patient sample described a homogeneous pain experience.

Use of the MPQ provided quantification of sensory, affective and evaluative components of pain in this group of patients with DPS. Values of MPQ subscales generated by this sample equaled or exceeded scores reported by patients with other pain syndromes, including menses, arthritis, cancer, phantom limb, toothache and childbirth $[19,20,29,31]$. Intercorrelations between $3 \mathrm{MPQ}$ scores suggested that most of the variance in the total pain rating index (PRI-T) was a result of the number of words chosen (NWC) and the affective pain rating index (PRI-A). A robust correlation between SPI-day and SPI-week suggested intrinsic stability of reported pain intensity. It may be argued that this stability resulted from insensitivity of the instrument to changes in perceived pain. However, Sternbach and co-workers $[44,45]$ demonstrated that the SPI measures small changes in pain intensity following surgical treatment of pain patients. Lack of significant relationships between SPI scores and MPQ subscales in the present investigation reflects the unidimensional nature of the SPI. Results of the Zung PAD index correlated with NWC of the MPQ. However, PAD scores demonstrated no relationships with other pain measures. In particular, the lack of association between PAD scores and PRI-A values was surprising because both tests ostensibly measure similar affective characteristics. This may indicate that the Zung Pain and Distress Scale measures variables in addition to pain quality, intensity and affective components.

Limitations in the applicability of these results to a large population of traumatic SCI patients with DPS are related to selection bias. The study group consisted of patients who met criteria for inclusion in a drug trial for the treatment of function-limiting dysesthetic pain syndrome. A small number of patients were not evaluated because of refusal to participate or inability to obtain transportation. In addition, results of the study cannot be extended to those traumatic SCI patients with DPS who do not have functional compromise as a result of their pain.

This investigation represents the first attempt to comprehensively and systematically describe the function-limiting dysesthetic pain syndrome following traumatic myelopathy. Patients with this syndrome are more likely to have incomplete sensory lesions, paraplegia, gunshot wounds to the spine and histories of non-surgical spinal stabilization. A variety of pharmacologic and non-pharmacologic treatments were used by this group without success. Pain quality and intensity, as measured by a number of established instruments, equaled or exceeded these previously reported for several other pain syndromes. It is clear that this syndrome has a profound impact upon the quality of life in a small but clinically significant group of SCI patients. 


\section{Acknowledgements}

This investigation was funded by a grant from the CNS Research Division of Bristol-Myers Corporation. Esther Stohl served as research assistant. Constance Keyserling, M.S., served as consultant for statistical analysis. Secretarial support was provided by Victoria Gregory and Patricia Wagner.

\section{References}

1 American Spinal Injury Association, Standards for Neurological Classification of Spinal Injury Patients, Amer. Spinal Injury Ass., Chicago, IL, 1982, pp. 2-14.

2 Banerjee, T., Transcutaneous nerve stimulation for pain after spinal injury, New Engl. J. Med., 291 (1974) 796.

3 Bedbrook, G.M., Pain in paraplegia and tetraplegia, In: G.M. Bedbrook (Ed.), Lifetime Care of the Paraplegic Patient, Churchill-Livingstone, Edinburgh, 1985, pp. 245-256.

4 Bors, E., Phantom limbs of patients with spinal cord injury, Arch. Neurol. Psychiat. (Chic.), 66 (1951) $610-631$.

5 Botterell, E.H., Callaghan, J.C. and Joussi, A.T., Pain in paraplegia: clinical management and surgical treatment, Proc. roy. Soc. Med., 47 (1953) 281-288.

6 Burke, D.C., Pain in paraplegia, Paraplegia, 10 (1973) 297-313.

7 Burke, D.C. and Woodward, J.M., Pain and phantom sensation in spinal paralysis. In: P.J. Vinken and G.W. Bruyn (Eds.), Handbook of Clinical Neurology, Vol. 26, Elsevier, New York, 1976, pp. $489-499$.

8 Cain, H.D., Subarachnoid phenol block in the treatment of pain and spasticity, Paraplegia, 3 (1965) $152-160$.

9 Crockett, D.J., Pricachin, K.M. and Craig, K.D., Factors of the language of pain in patient and volunteer groups, Pain, 4 (1977) 175-182.

10 Davis, L., Treatment of spinal cord injuries, Arch. Surg., 69 (1954) 488-495.

11 Davis, L. and Martin, J., Studies upon spinal cord injuries. II. The nature and treatment of pain, J. Neurosurg., 4 (1947) 483-491.

12 Davis, R., Pain and suffering following spinal cord injury, Clin. Orthop. relat. Res., 112 (1975) 76-80.

13 Davis, $R$, and Lentini, $R$., Transcutaneous nerve stimulation for treatment of pain in patients with spinal cord injury, Surg. Neurol., 4 (1975) 100-101.

14 De Saussure, R.L., Lateral spinothalamic tractotomy for relief of pain in cauda equina injury, Arch. Neurol. Psychiat. (Chic.), 64 (1950) 708-714.

15 Donovan, W.H., Dimitrijevic, M.R., Dahm, L. and Dimitrijevic, M., Neurophysiological approaches to chronic pain following spinal cord injury, Paraplegia, 20 (1982) 135-146.

16 Druckman, R. and Lende, R., Central pain of spinal cord origin: pathogenesis and surgical relief in one patient, Neurology (Minneup.), 15 (1965) 518-522.

17 Dubuisson, D. and Melzack, R., Classification of clinical pain descriptions by multiple group discriminant analysis, Exp. Neurol., 51 (1976) 480-487.

18 Freeman, L.W. and Hamburger, R.F., Surgical relief of pain in paraplegic patients, Arch. Surg., 55 (1947) 433-440.

19 Graham, C., Bond, S.S., Gerkovich, M.M. and Cock, M.R., Use of the McGill Pain Questionnaire in the assessment of cancer pain: replicability and consistency, Pain, 8 (1980) 377-387.

20 Grushka, M. and Sessle, B.J., Applicability of the McGill Pain Questionnaire to the differentiation of 'toothache' pain, Pain, 19 (1984) 49-57.

21 Grzesiak, R.C., Relaxation techniques in treatment of chronic pain, Arch. phys. Med. Rehab., 58 (1977) $270-272$.

22 Guttmann, L., Disturbances of sensibility. In: L. Guttmann (Ed.), Spinal Cord Injuries: Comprehensive Management and Research, 2nd edition, Blackwell Scientific Publications, Oxford, 1973, pp. $280-305$. 
23 Heilporn, A., Two therapeutic experiments on stubborn pain in spinal cord lesions: coupling melitracen-flupenthixen and the transcutaneous nerve stimulation, Paraplegia, 15 (1977-78) 368-372.

24 Kaplan, L.I., Grynbaum, B.B., Floyd, K.E. and Rusk, H.A., Pain and spasticity in patients with spinal cord dysfunction: results of a follow-up study, J. Amer. med. Ass., 182 (1962) 918-925.

25 Kennedy, R.H., The new viewpoint toward spinal cord injuries, Ann. Surg., 124 (1946) 1057-1065.

26 Klepac, R.K., Dowling, J., Rokke, P., Dodge, L. and Schafer, L., Interview vs. paper-and-pencil administration of the McGill Pain Questionnaire, Pain, 11 (1981) 241-246.

27 Krueger, E.G., Management of painful states in injuries of the spinal cord and cauda equina, Amer. J. phys. Med., 39 (1960) 103-110.

28 Maury, M., About pain and its treatment in paraplegics, Paraplegia, 15 (1977-78) 349-352.

29 Melzack, R., The McGill Pain Questionnaire: major properties and scoring methods, Pain, 1 (1975) $277-299$.

30 Melzack, R. and Loeser, J.D., Phantom body pain in paraplegics: evidence for a central "pattern generating mechanism' for pain, Pain, 4 (1978) 195-210.

31 Melzack, R., Taenzer, P., Feldman, P. and Kirch, R.A., Labour is still painful after prepared childbirth training, Canad. med. Ass. J., 125 (1981) 357-363.

32 Michaelis, L.S., The problem of pain in paraplegia and tetraplegia, Bull. N.Y. Acad. Med., 46 (1970) $88-96$.

33 Midwest Regional Spinal Cord Injury Case System Data Base, 1985.

34 Munro, D., Rehabilitation of veterans paralyzed as the result of injury to the spinal cord and cauda equina, Amer. J. Surg., 75 (1948) 3-17.

35 Munro, D., Two-year end-results in the total rehabilitation of veterans with spinal-cord and cauda-equina injuries, New Engl. J. Med., 242 (1950) 1-10.

36 Nashold, B.S. and Bullitt, E., Dorsal root entry zone lesions to control central pain in paraplegics, J. Neurosurg., 55 (1981) 414-419

37 Nepomuceno, C., Fine, P.R., Richards, J.S., Gowens, H., Stover, S.L., Rantanuabol, U. and Houston. R., Pain in patients with spinal cord injury, Arch. phys. Med. Rehab., 60 (1979) $605-609$.

38 Pollock, L.I., Brown, M., Boshes, B., Finkelman, I, Chor, H., Arieff, A.J. and Finkle, J.R., Pain below the level of injury of the spinal cord, Arch. Neurol. Psychiat. (Chic.), 65 (1951) 319-322.

39 Porter, R.W., Hohmonn, G.W., Bors, E. and French, J.D., Cordotomy for pain following cauda equina injury, Arch. Surg., 92 (1966) 765-770.

40 Prieto, E.J., Hopson, L., Bradley, L.A., Byrne, M., Geisinger, K.F., Midax, D. and Marchisello, P.J. The language of low back pain: factor structure of the McGill Pain Questionnaire, Pain, 8 (1980) $11-19$

41 Richards, J.S., Meredith, R.L., Nepomuceno, C., Fine, P.R. and Bennett, G., Psycho-social aspects of chronic pain in spinal cord injury, Pain, 8 (1980) 355-366.

42 Richardson, R.R. Meyer, P.R. and Cerullo, L.J., Neurostimulation in the modulation of the intractable paraplegic and traumatic neuroma pains, Pain, 8 (1980) 75-84.

43 Richter, H.P. and Seitz, K., Dorsal root entry zone lesions for the control of deafferentation pain: experience in ten patients, Neurosurgery, 15 (1984) $956-959$.

44 Sternbach, R.A., Pain Patients - Traits and Treatment, Academic Press, New York, 1974.

45 Sternbach, R.A., Murphy R.W., Timmermans, G., Greenhoot, J.H. and Akeson, W.H., Measuring the severity of clinical pain. In: J.J. Bonica (Ed.), Advances in Neurology, Vol. 4, Raven Press, New York, 1974. pp. $281-288$

46 Van Buren, J. and Kleinknecht, R.A., An evaluation of the McGill Pain Questionnaire for use in dental pain assessment, Pain, 6 (1979) 23-33.

47 Waisbrod. H., Hansen, D. and Gerbershagen, H.U., Chronic pain in paraplegics, Neurosurgery, 15 (1984) $933-934$

48 Young, J.S., Burns, P.E., Bowen, A.M. and McCutchen, R., Spinal Cord Statistics: Experience of the Regional Spinal Cord Injury Systems, Good Samaritan Medical Center, Phoenix, AZ, 1982.

49 Zankel, H.T., Sutton, B.B. and Burney, T.E., A paraplegic program under physical medicine and rehabilitation: one year's experience, Arch. phys. Med. Rehab., 35 (1954) 296-302.

50 Zung. W.M., A self-rating pain and distress scale, Psychosomatics, 24 (1983) 887-894. 